\title{
Diagnostic utility of TP53 and cytokeratin 7 immunohistochemistry in idiopathic inflammatory bowel disease-associated neoplasia
}

Hao Xie ${ }^{1,2}$, Shu-Yuan Xiao ${ }^{3}$, Rish Pai ${ }^{1}$, Wei Jiang ${ }^{1}$, Bonnie Shadrach ${ }^{1}$, Paula Carver ${ }^{1}$, Yinghong Wang ${ }^{4}$, Bo Shen ${ }^{4}$, Weixun Zhou ${ }^{5}$ and Xiuli Liu ${ }^{1}$

${ }^{1}$ Department of Anatomic Pathology, Cleveland Clinic, Cleveland, OH, USA; ${ }^{2}$ Cleveland Clinic Lerner College of Medicine of Case Western Reserve University, Cleveland, OH, USA; ${ }^{3}$ Department of Pathology, University of Chicago, Chicago, IL, USA; ${ }^{4}$ Department of Gastroenterology, Digestive Disease Institute, Cleveland Clinic, Cleveland, OH, USA and ${ }^{5}$ Department of Pathology, Peking Union Medical College Hospital, Chinese Academy of Medical Sciences, Beijing, China

Long-standing inflammatory bowel disease is associated with increased risk of developing colorectal adenocarcinoma. Significant intra- and inter-observers' variability exists in histologic interpretation of dysplasia in surveillance biopsies. In this study, we evaluated the utility of a panel of immunohistochemical markers in diagnosing inflammatory bowel disease-associated neoplasia. We reviewed 39 colectomy specimens with inflammatory bowel disease-associated neoplasia. In these 39 cases, we identified 172 foci of interest ( 5 normal, 58 negative for dysplasia, 15 indefinite for dysplasia, 59 low-grade dysplasia, 18 highgrade dysplasia, and 17 invasive adenocarcinoma). They were subjected to immunohistochemistry for TP53 and CK7. Logistic regression was used to evaluate their association with the presence of dysplasia. Receiver operating characteristic curves were used to determine the optimal cutoffs and assess the diagnostic performance of TP53 and CK7. Both TP53 nuclear staining and CK7 immunoreactivity gradually increased in the progression of inflammatory bowel disease-associated neoplasia $(P<0.0001)$. CK7 immunoreactivity increased along with the increase of inflammation severity $(P=0.0002)$ as well as reactive changes $(P=0.04)$ in the colonic mucosa. But TP53 nuclear staining was independent of either feature. When both TP53 $>8 \%$ and CK7 $>30 \%$ as identified from logistic regression and receiver operating characteristic curves were used to diagnose dysplasia, the specificity achieved as high as $95 \%$. When either TP53 $>8 \%$ or CK7 $>30 \%$ was used to diagnose dysplasia, the sensitivity achieved was $82 \%$. Our results suggested that a combination of CK7 and TP53 immunohistochemistry may be helpful in diagnosing inflammatory bowel disease-associated dysplasia in difficult cases.

Modern Pathology (2014) 27, 303-313; doi:10.1038/modpathol.2013.133; published online 26 July 2013

Keywords: Crohn's disease; cytokeratin 7; dysplasia; inflammatory bowel disease; neoplasia; TP53; ulcerative colitis

Long-standing inflammatory bowel disease, either ulcerative colitis or Crohn's disease, is associated with an increased risk of developing colorectal adenocarcinoma. Regular colonoscopic surveillance for the detection of dysplasia is recommended in

Correspondence: Dr X Liu, MD, PhD, Department of Anatomic Pathology, Cleveland Clinic, 9500 Euclid Avenue/L25, Cleveland, $\mathrm{OH} 44195$, USA.

E-mail: liux3@ccf.org

Received 22 October 2012; revised 30 May 2013; accepted 31 May 2013; published online 26 July 2013 long-standing inflammatory bowel disease and histologic diagnosis of dysplasia remains the best marker for the presence of synchronous/metachronous dysplasia or adenocarcinoma. ${ }^{1}$ However, significant intra- and inter-observer variability exists in histologic interpretation of dysplasia in colon biopsy. ${ }^{2}$ The diagnostic accuracy may increase by using a biomarker-based surveillance strategy but such biomarkers with good testing characteristics currently do not exist.

Similar to sporadic colorectal adenocarcinoma, the development of colitis-associated colorectal 
adenocarcinoma, is hypothesized to involve a multistep process affecting multiple genes. However, the underlying tumorigenesis of colitis-associated colorectal adenocarcinoma is different from that of sporadic colorectal adenocarcinoma. ${ }^{3}$ This was demonstrated by the distinct histomorphology of colitis-associated colorectal adenocarcinoma. ${ }^{4}$ In addition, our previous study on ileal pouch and peri-pouch adenocarcinomas developed in inflammatory bowel disease patients showed that approximately $60 \%$ of these tumors expressed CK7, rarely had nuclear $\beta$-catenin accumulation, ${ }^{5}$ in contrast to the pattern seen in sporadic colorectal adenocarcinoma. ${ }^{6}$ Therefore, CK7 expression may be associated with tumorigenesis in inflammatory bowel disease.

In line with our previous findings, overexpression of CK7 was also reported in a subgroup of colitisassociated colorectal adenocarcinoma, namely, lowgrade glandular adenocarcinoma ${ }^{7}$ and in preneoplastic and neoplastic lesions in ulcerative colitis, ${ }^{8}$ suggesting a potential use of this marker in diagnosing colitis-associated dysplasia and predicting progression of colitis-associated colorectal adenocarcinoma. However, these studies had small sample size and thus mainly relied on descriptive analysis, whose conclusions might have suffered from the presence of potential confounders. In this case, the expression of CK7 has not been previously examined for its association with the presence of inflammation ${ }^{9}$ or reactive changes, ${ }^{1}$ two common factors profoundly interfering with the interpretation of dysplasia.

In addition to CK7, other markers were also studied for their predictive role in the development of colitis-associated colorectal adenocarcinoma. TP53 nuclear immunoreactivity is one of the most promising candidates. ${ }^{1,10}$ Nuclear TP53 expression has been reported to be predictive of colitis-associated colorectal adenocarcinoma, ${ }^{11}$ in addition to DNA ploidy, or combined with $\alpha$-methylacyl coenzyme A racemase immunoreactivity. ${ }^{12}$ However, TP53 immunoreactivity was only noted in no more than $50 \%$ of inflammatory bowel disease-associated neoplasia. ${ }^{13}$ Combination of TP53 nuclear immunoreactivity with a complimentary marker, such as CK7, may increase its diagnostic performance.

The purpose of this study was to examine the expression of CK7 and TP53 in a spectrum of well defined and centrally reviewed epithelial changes along the pathway of inflammatory bowel diseaseassociated neoplasia. Confounders such as the presence of inflammatory and reactive epithelial changes were also analyzed.

\section{Materials and methods}

\section{Case Identification}

From the pathology files of Cleveland Clinic, we identified 39 patients who underwent colectomy for inflammatory bowel disease from 1991 to 1997 in order to ensure adequate follow-up. The histologic sections were reviewed by three expert GI pathologists (XL, RP, and S-YX). Eligible lesions included low-grade dysplasia, high-grade dysplasia, and invasive adenocarcinomas. In addition, some normal colonic mucosa, mucosa involved by inflammatory bowel disease but negative for dysplasia, and mucosa involved by inflammatory bowel disease and with epithelial changes indefinite for dysplasia from the resected specimens were also included.

\section{Histopathological Evaluation}

The diagnostic criteria for chronic colitis include a combination of two of the following three features: crypt/architectural distortion, presence of basal lymphoplasmacytosis, and metaplasia (pseudopyloric gland metaplasia at any portion of the colon and rectum; Paneth cell metaplasia when the section taken is distal to the splenic flexure). Dysplasia was evaluated according to the previously published criteria $^{14}$ (Figure 1). For difficult cases, three gastrointestinal pathologists (XL, RP, and S-YX) reviewed the slides independently and a consensus diagnosis (agreed by at least two pathologists) was used. For cases that could not be agreed on, an 'indefinite for dysplasia' diagnosis was assigned. All 17 adenocarcinoma cases were invasive at least into the submucosa. All histopathological materials were fixed in Hollande's fixative and embedded in paraffin.

The presence of inflammation in each focus of interest was graded as none, mild (when there is mild increase in mononuclear cells in the lamina propria with rare neutrophils), moderate (when there is expansion of the lamina propria by mononuclear cells with cryptitis and crypt abscess), and severe (when there is marked basal lymphoplasmacytosis and significant neutrophilic inflammation, which may result in ulceration). In our analysis, the groups with none and mild inflammation were combined as the low inflammation group; and the moderate and severe inflammation groups were combined as high inflammation group. Further, the epithelial cells in each non-dysplastic focus (normal, diseased mucosa but negative for dysplasia, and diseased mucosa indefinite for dysplasia) were evaluated for the presence of reactive changes using a semi-quantitative scale as follows: none (no reactive change), mild (slight loss of surface mucin, slightly increased nuclear to cytoplasmic (N/C) ratio, and mild nuclear disarray), moderate (more pronounced loss of mucin with regenerative changes in the lower half of the crypts), and severe (pronounced loss of mucin with regenerative changes involving the entire length of the crypts). ${ }^{14}$ In our analysis, the absent and mild reactive change groups were combined as low reactive change group; the moderate and marked reactive change groups were combined as high reactive 
a
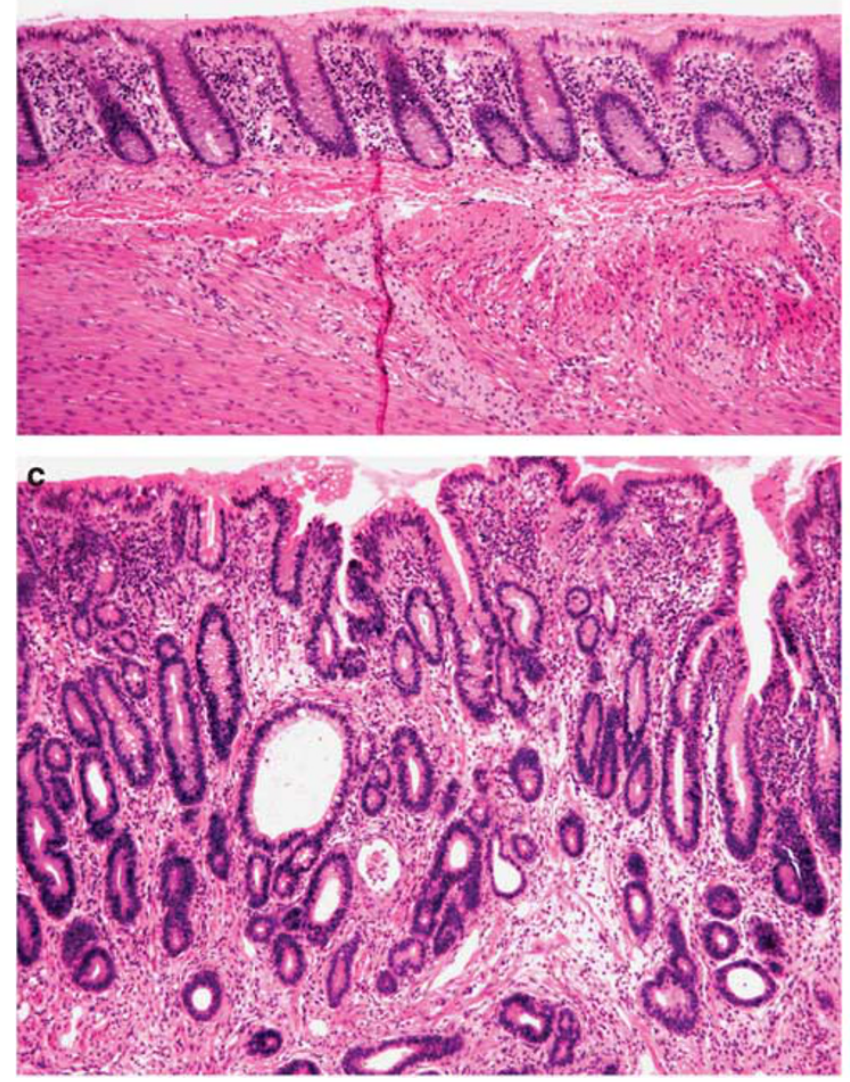
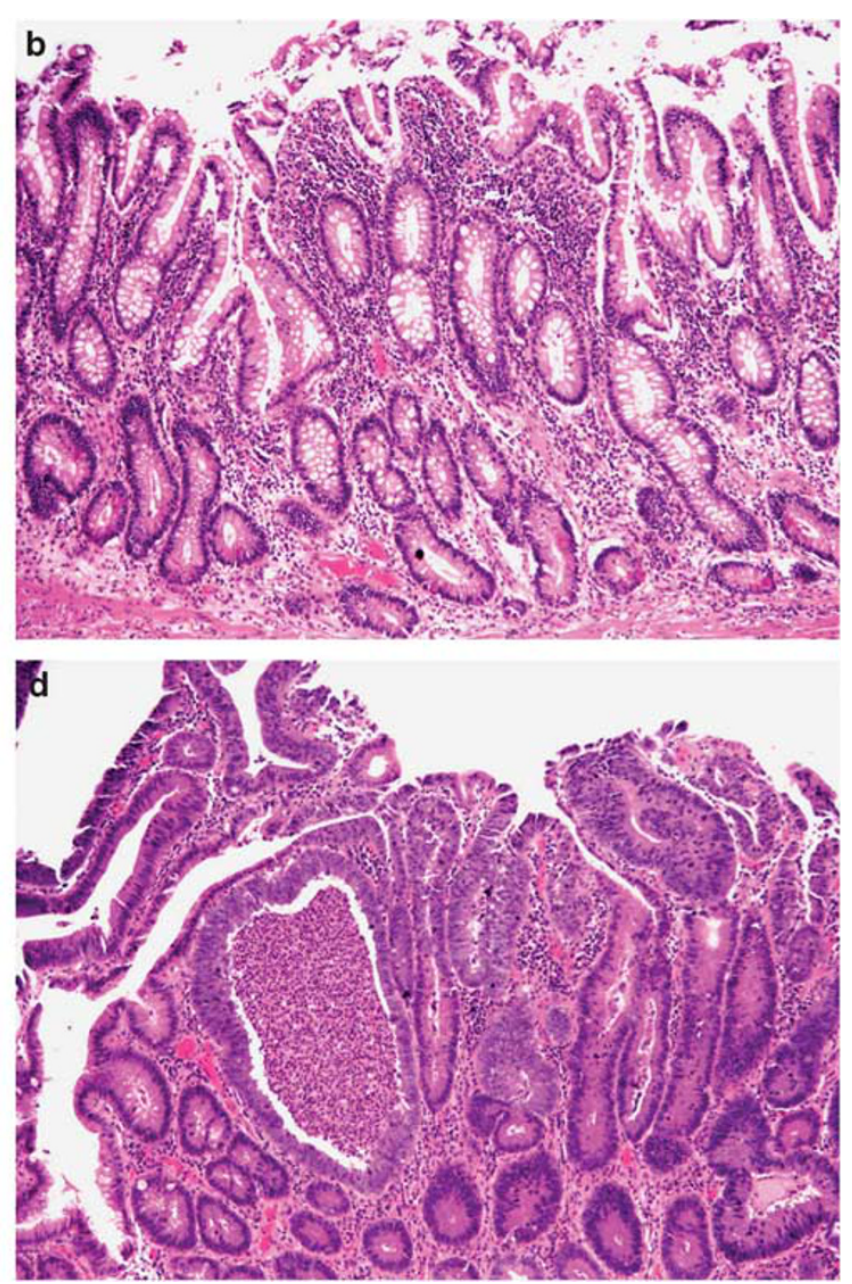

Figure 1 Representative images of normal colon (a, H\&E stain, $\times 100$ ), chronic colitis, negative for dysplasia (b, H\&E stain, $\times 100)$, chronic colitis with low-grade dysplasia $(\mathbf{c}, \mathrm{H} \& \mathrm{E}$ stain, $\times 100)$, and chronic colitis with high-grade dysplasia (d, H\&E stain, $\times 100)$.

change group. Representative degrees of inflammation and reactive changes are shown in Figures 2 and 3 , respectively.

\section{Immunohistochemistry}

Immunohistochemical staining for TP53 and CK7, was performed on whole tissue section from Hollande's fixed and paraffin-embedded tissue. Briefly, de-paraffinized tissue sections were stained with antibodies against TP53 (clone DO-7, at 1:20 working dilution; Dako, Carpinteria, CA, USA), CK7 (clone OV-TL 12/30, diluted 1:40; Dako), diaminobenzidine served as the chromogen. Positive and negative controls for these antibodies were included for the study. The antigen retrieval step for slides to be stained for TP53 consisted of CC1 (Tris/Borate/EDTA buffer, pH 8.0-8.5; Ventana Medical Systems, Tuscon, AZ, USA) for $8 \mathrm{~min}$ at $95^{\circ} \mathrm{C}, 28 \mathrm{~min}$ at $100^{\circ} \mathrm{C}$, and then an $8 \mathrm{~min}$ cool down to $37^{\circ} \mathrm{C}$. The antigen retrieval step for slides to be stained for CK7 consisted of Protease 1 (Ventana Medical Systems) for 4 min at $37^{\circ} \mathrm{C}$.
A total of 172 foci of interest were selected for immunohistochemical study for TP53 and CK7. Of these foci, 5 (3\%) had normal histology, 58 (34\%) with colitis but negative for dysplasia, $15(9 \%)$ with colitis and indefinite for dysplasia, 59 (34\%) with low-grade dysplasia, 18 (10\%) with high-grade dysplasia, and $17(10 \%)$ with invasive adenocarcinoma. All immunohistochemical stains were evaluated by one pathologist (XL). The presence or absence of nuclear staining was evaluated in the tissue of whole section for TP53. The expression of TP53 was determined as percentage of cells within a high-power field showing strong nuclear staining (Figure 4a). CK7 expression was determined as percentage of cells with membranous/cytoplasmic staining (Figure 4b).

\section{Statistical Analysis}

Univariate analysis was used to describe cases with different histopathological diagnoses. CK7, TP53, and inflammation severity were included as 


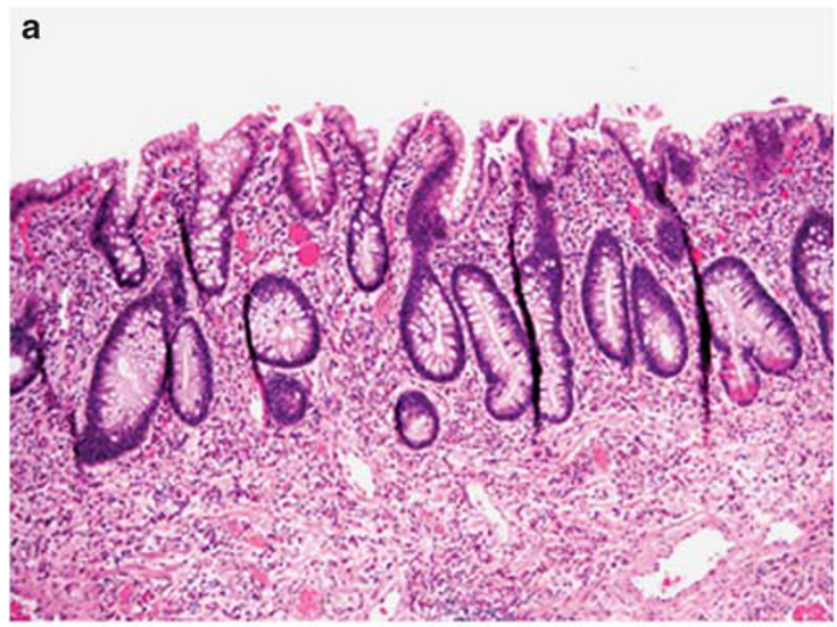

b
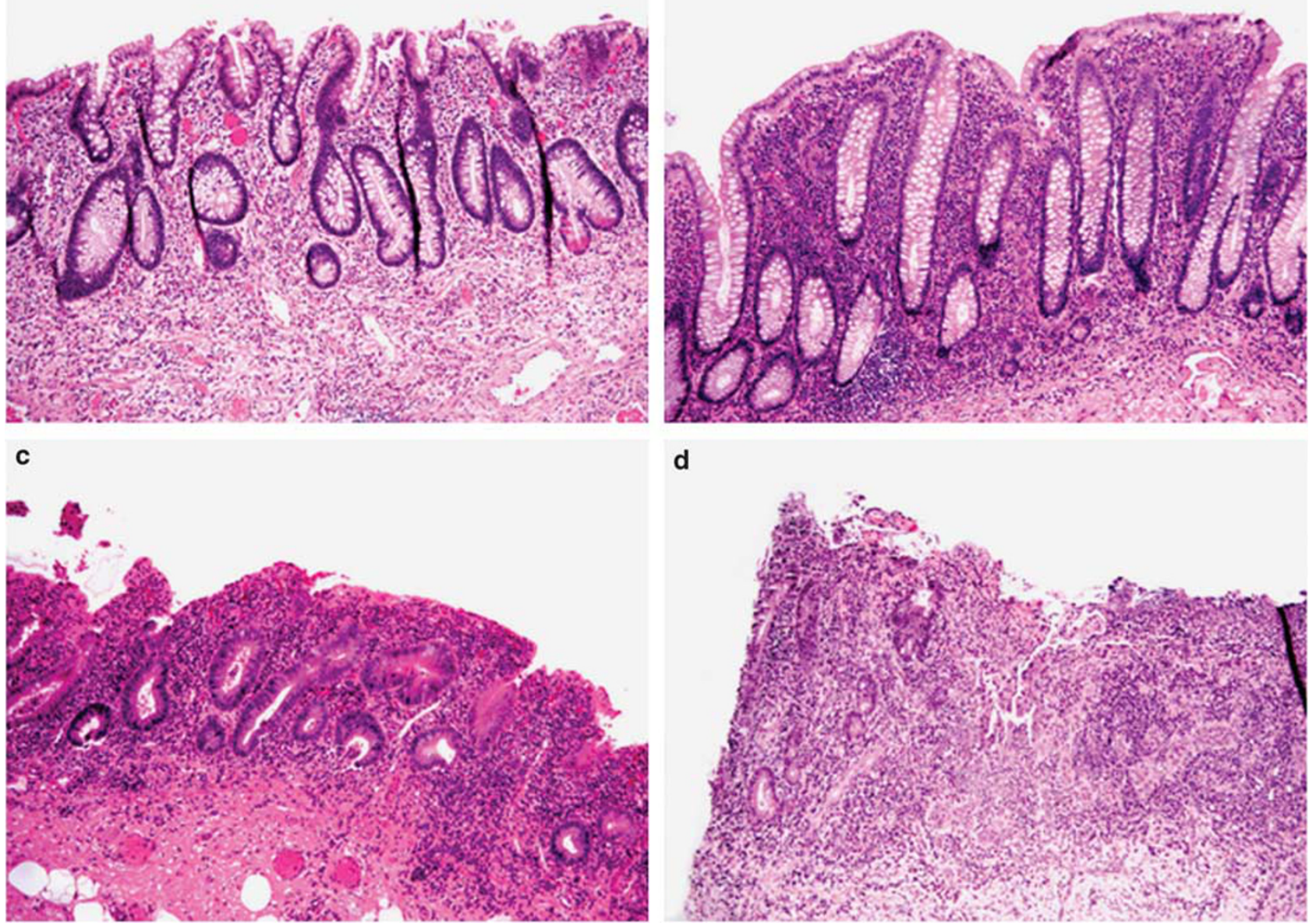

d

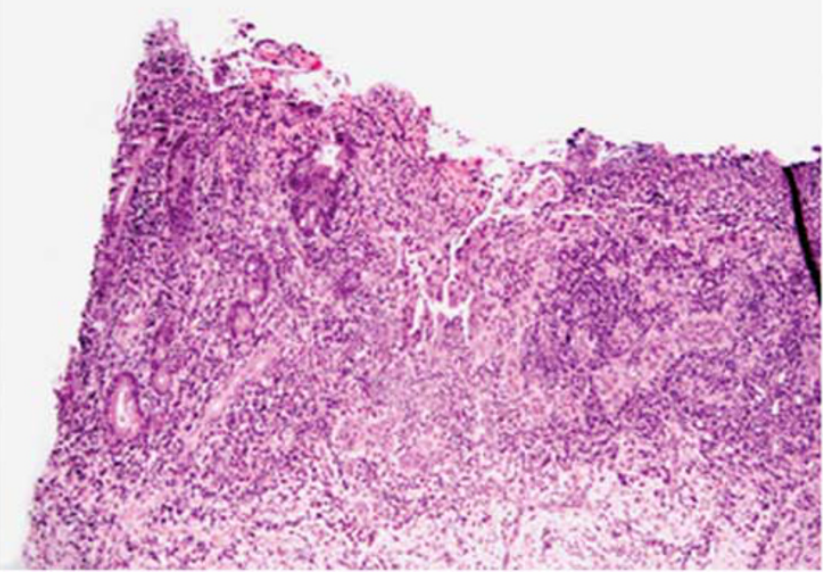

Figure 2 Representative images of inflammation from none (a, H\&E stain, $\times 100)$, mild (b, H\&E stain, $\times 100)$, moderate $(\mathbf{c}$, H\&E stain, $\times 100$ ), and severe (d, H\&E stain, $\times 100)$.

independent variables in a multivariable logistic regression model. The two levels of dependent variable include non-dysplasia and dysplasia. The former included negative and indefinite cases. The latter included low-grade and high-grade dysplasia. In the sensitivity analysis, the non-dysplasia group included negative cases only; the dysplasia group did not change. The forward/backward model selection based on Bayesian information criterion was used for the initial logistic model with three main effects and their two-way interactions to identify variables independently associated with the presence or absence of dysplasia. All logistic regression results were presented as odds ratio, 95\% confidence interval (CI), and corresponding $P$-value. Multicollinearity was evaluated with variance inflation factor. Model diagnostics included effect plots, influence plots, and residuals. We evaluated the goodness of fit of logistic regression models using receiver operating characteristic curves and reported area under the curve. All the models were subjected to internal validation using bootstrapping. Continuous data were analyzed using one-way ANOVA.
A $P$-value $<0.05$ was considered statistically significant. All the statistical tests were two-sided. All statistical analyses were performed using R software package (R Development Core Team, 2012; R Foundation for Statistical Computing, Vienna, Austria).

\section{Results}

\section{Demographic and Clinical Features}

A total of 39 patients (14 women and 25 men) were included in this study. The median age at colectomy was 40 years (range: 17-76 years). All of them had colectomy for neoplasia arising from inflammatory bowel disease including 31 ulcerative colitis patients and 8 Crohn's disease patients. The highest grade of neoplasia diagnosed in their colectomy specimens was high-grade dysplasia in 12 patients and invasive adenocarcinoma in 27 patients including 21 cases with unifocal adenocarcinoma and 6 with multifocal cancer. The diagnosis of inflammatory bowel disease was confirmed by clinical, 

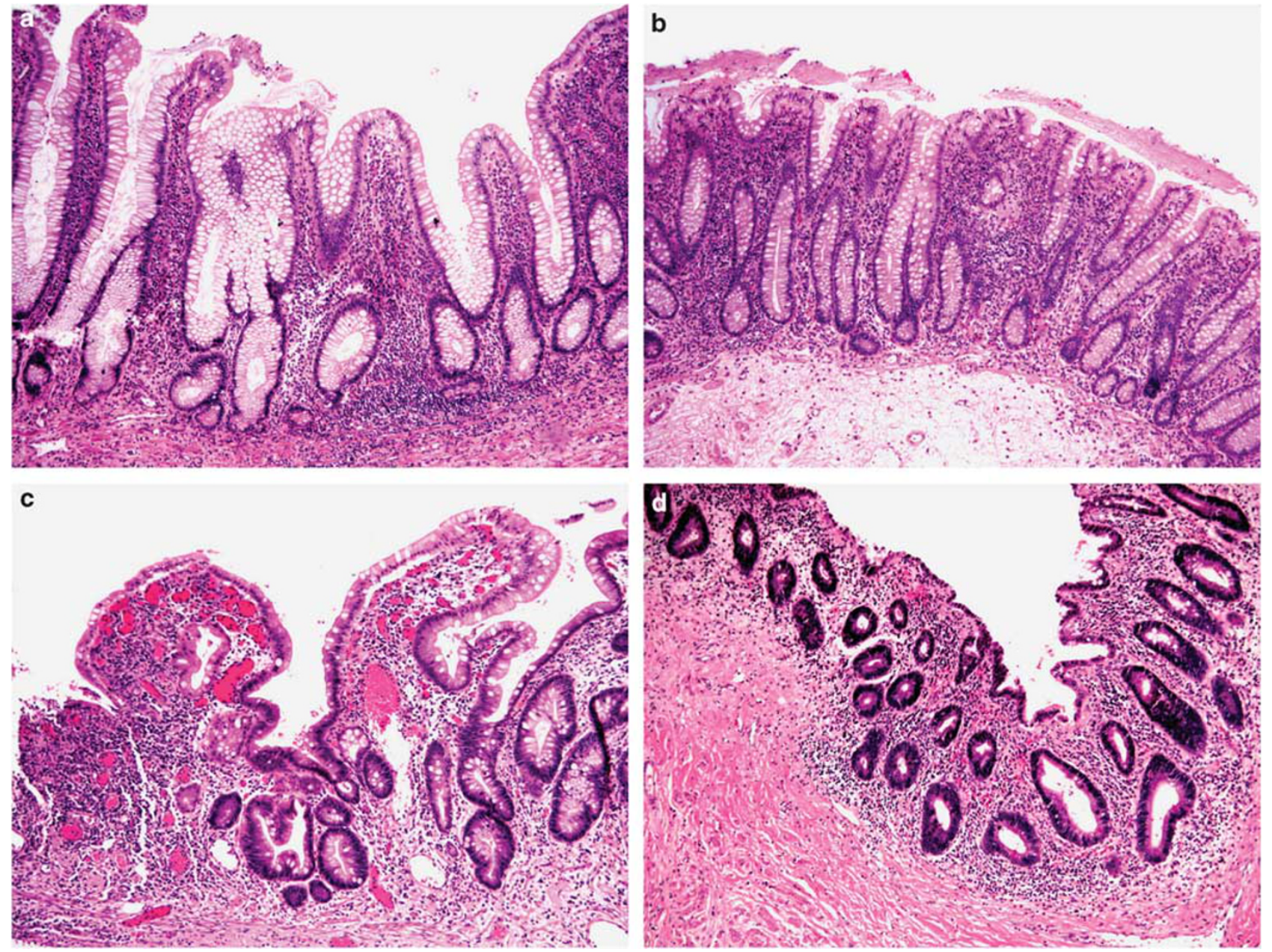

Figure 3 Representative images of reactive changes in cases either negative for dysplasia or indefinite for dysplasia. (a) no reactive changes (H\&E stain, $\times 100)$. (b) Mild reactive changes (H\&E stain, $\times 100)$. (c) Moderate reactive changes (H\&E stain, $\times 100) .(\mathbf{d})$ Marked reactive changes $(\mathrm{H} \& \mathrm{E}$ stain, $\times 100)$.

endoscopic, and in some cases, radiographic features, along with histologic evaluation of the colectomy specimen.

\section{Association between Nuclear TP53 Expression and Inflammation and Reactive Changes in Inflammatory Bowel Disease-Associated Neoplasia}

The mean nuclear expression of TP53 was 26\% (95\% CI: $20-31 \%$ ). As shown in Figure 5a, TP53 nuclear expression increased along with the progression of the inflammatory bowel disease-associated neoplasia $(P<0.0001)$ from $<1 \%$ in normal colon to $57 \%$ in invasive adenocarcinoma. Specifically, the mean nuclear TP53 expression in nondysplastic colitis group of 73 foci (negative and indefinite for dysplasia) was significantly lower than that in low-grade and high-grade dysplasia group of 77 foci ( $6 \%$ vs $39 \%, P<0.0001$ ).

We examined and plotted the relationship between TP53 nuclear expression and inflammation
(Figure 5b) and reactive changes (Figure 5c). The expression of TP53 was independent of the severity of inflammation $(P=0.5)$. Similarly, the expression of TP53 was independent of the degree of reactive changes $(P=0.1)$. In the subset of foci with negative or indefinite dysplasia, the expression of TP53 was also independent of the severity of inflammation (6\% in low inflammation group of 43 foci vs $6 \%$ in high inflammation group of 30 foci, $P=0.9$ ) and reactive changes $(8 \%$ in low reactive changes group of 39 foci vs $4 \%$ in high reactive changes group of 34 foci, $P=0.2$ ).

\section{Association between CK7 Expression and Inflammation and Reactive Changes in Inflammatory Bowel Disease-Associated Neoplasia}

The mean percentage of CK7 expressing cells was 42\% (95\% CI: 36-49\%). As shown in Figure 6a, CK7 expression increased along with the progression of the inflammatory bowel disease-associated neoplasia 


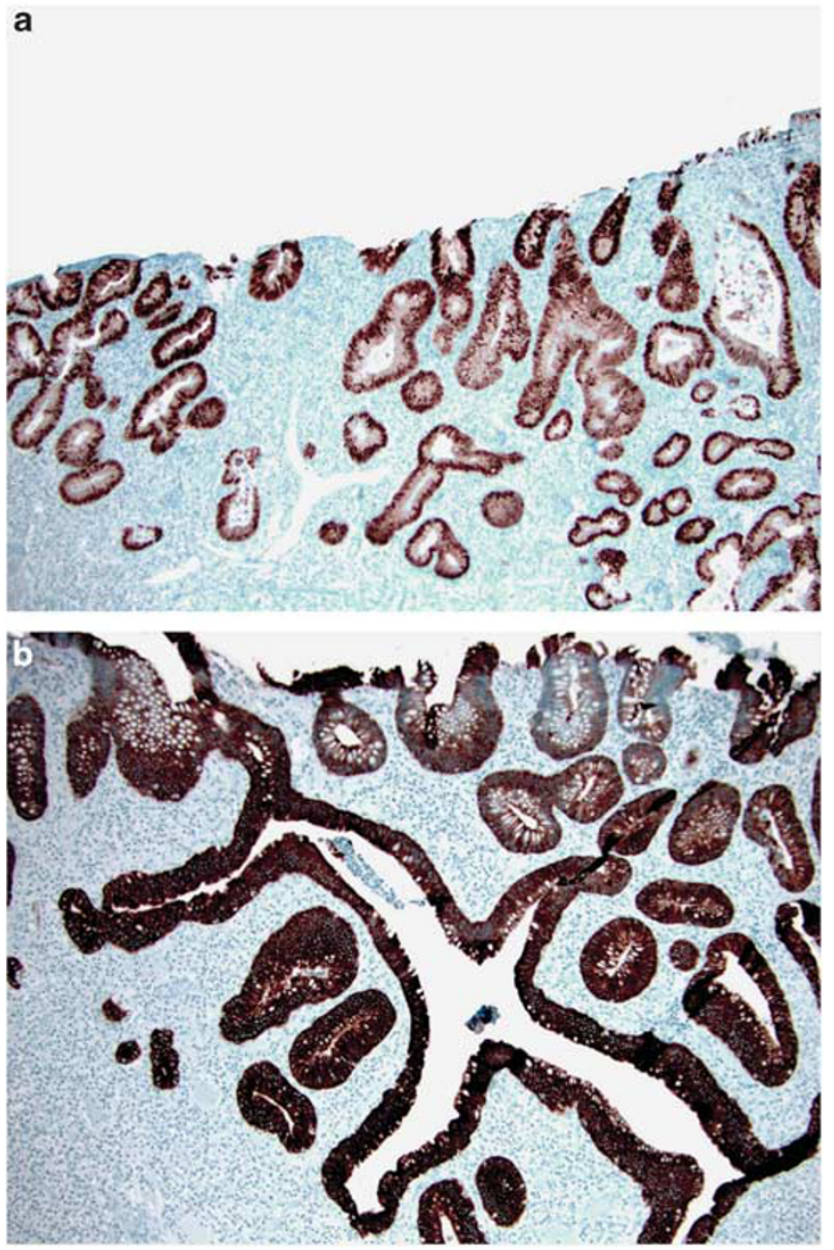

Figure 4 Representative images of positive TP53 nuclear staining (a, immunoperoxidase stain, $\times 100$ ) and positive CK7 expression (b, immunoperoxidase stain, $\times 100$ ).

$(P<0.0001)$ from $2.2 \%$ in normal colon to $69 \%$ in invasive adenocarcinoma. Specifically, the mean CK7 expression in the nondysplastic colitis group of 73 foci (negative and indefinite for dysplasia) was significantly lower than that in low-grade and high-grade dysplasia group of 77 foci $(28 \%$ vs $53 \%$, $P=0.0002$ ).

We examined and plotted the association between CK7 expression and inflammation (Figure 6b) and reactive changes (Figure 6c). The expression of CK7 increased along with the severity of inflammation $(P=0.0002)$ from $26 \%$ in the inflammationabsent group to $75 \%$ in the severe inflammation group. Similarly, the expression of CK7 increased along with the degree of reactive changes $(P=0.04)$ from $13 \%$ in the absence of reactive changes group to $61 \%$ in marked reactive changes group. However, in the subset of foci with negative or indefinite for dysplasia, the expression of CK7 was not associated with the severity of inflammation $26 \%$ in the low inflammation group of 43 foci vs $31 \%$ in the high inflammation group of 30 foci, $P=0.5$ ). But its positive association with reactive changes $(18 \%$ in low reactive changes group of 39 foci vs $39 \%$ in the high reactive change group of 34 foci, $P=0.02$ ) was still present.

\section{Logistic Regression Model for the Risk Factors of Dysplasia}

We evaluated the association between TP53 and CK7 expression, inflammation, and the presence of dysplasia using a multivariable logistic regression model (Table 1). The full models included both three main effects and their two-way interactions. As summarized in Table 1, both TP53 and CK7 were significant contributors to the prediction of dysplasia $(P=0.02$ and $P=0.04$, respectively). There was $3 \%$ increase in the odds of the presence of dysplasia per 1\% increase of TP53 expression, while adjusting for the presence of other variables. Similarly, there was $1 \%$ increase in the odds of the presence of dysplasia per $1 \%$ increase of CK7 expression, while adjusting for the presence of other variables. There is a trend for inflammation to be a significant contributor to the prediction of dysplasia $(P=0.06)$. No significant two-way interactions among TP53, CK7, and inflammation were present. The full model was then subjected to a step-wise model selection. The reduced model only identified TP53 and CK7 as variables significantly associated with the presence of dysplasia $(P<0.0001$ and $P=0.001$, respectively).

\section{The Utility of TP53 and CK7 in the Diagnosis of Dysplasia}

Receiver operating characteristic curves were used to assess the goodness of fit of logistic regression models with TP53, CK7, or a combination of TP53 and CK7 as independent variables (Figure 7). The area under the curve was reported in Table 2. A combination of TP53 and CK7 as diagnostic markers achieved area under the curve 0.81 , sensitivity $68 \%$, specificity $85 \%$, positive predictive value $82 \%$, and negative predictive value $71 \%$ at the prevalence of dysplasia (51\%) in this study cohort. We also identified the optimal cutoff value of $8 \%$ and $30 \%$ for the use of TP53 and CK7 dichotomization, respectively. Of the total number of foci, 68 (40\%) foci had high expression of TP53, $104(60 \%)$ foci had low expression of TP53, 83 (48\%) foci had high expression of CK7, and 89 (52\%) foci had low expression of CK7. The frequency of positive TP53 (defined as nuclear immunoreactivity in $>8 \%$ cells) significantly increased along with the progression of neoplasia $(P<0.0001)$ : normal $0(0 \%)$ focus, negative $7(12 \%)$ foci, indefinite for dysplasia $5(33 \%)$ foci, low-grade dysplasia $33(56 \%)$ foci, high-grade dysplasia 11 (61\%) foci, and cancer 12 $(71 \%)$ foci. The frequency of positive CK7 (defined as immunoreactivity in $>30 \%$ cells) significantly increased along with the progression of neoplasia $(P=0.001)$ : normal $0(0 \%)$ focus, negative $18(31 \%)$ 

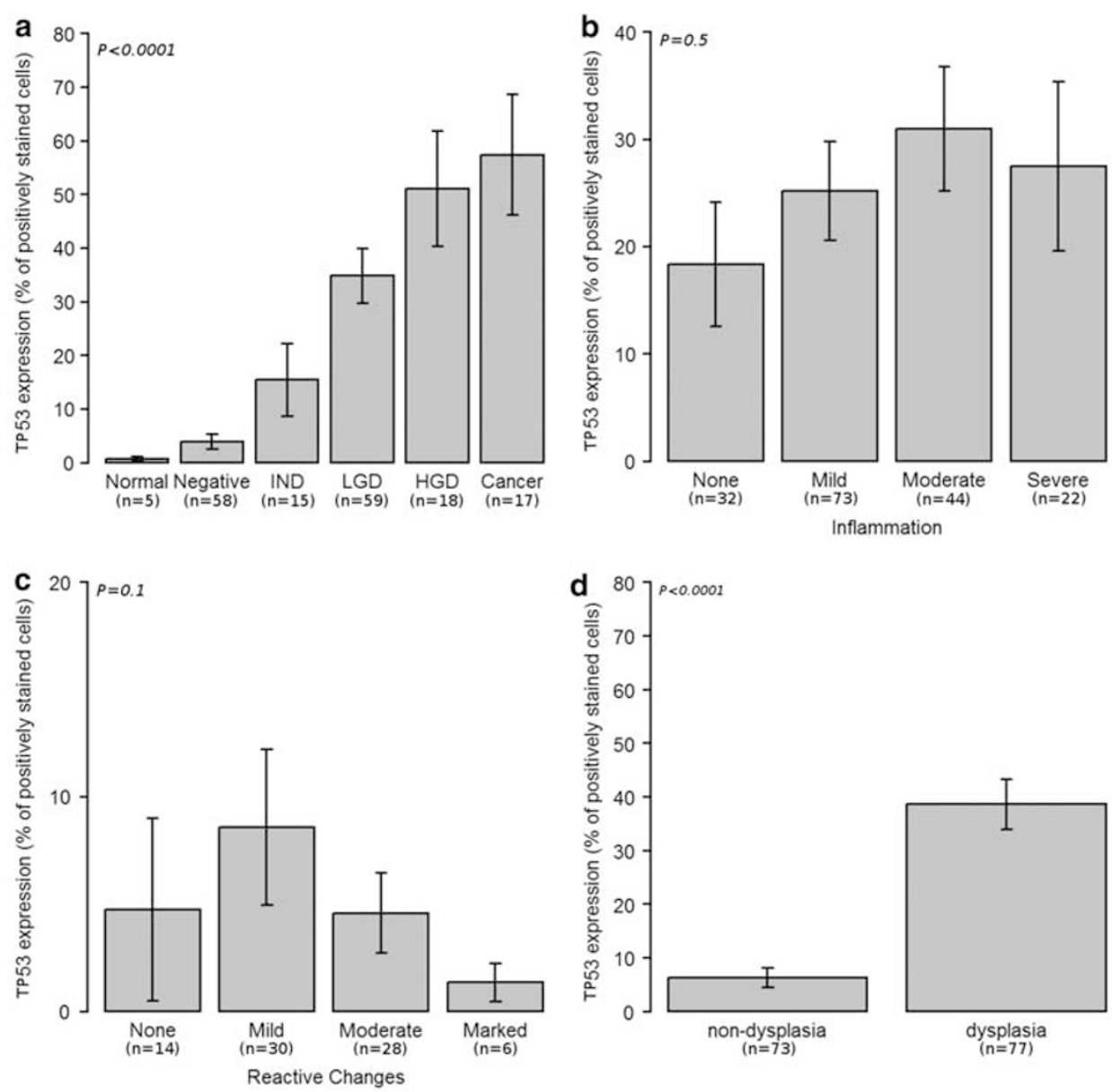

Figure 5 TP53 expression (\% of positively stained nuclei) and its relationship with histologic neoplasia diagnosis (a), the presence of inflammation (b), reactive changes (c), and dysplasia diagnosis (d). HGD, high-grade dysplasia, IND, indefinite for dysplasia; LGD, lowgrade dysplasia. Error bar: 1 s.e.

foci, indefinite for dysplasia 6 (40\%) foci, low-grade dysplasia $36(61 \%)$ foci, high-grade dysplasia 11 $(61 \%)$ foci, and cancer $12(71 \%)$ foci. For example, dysplastic foci with positive but relatively low TP53 expression and negative CK7 expression were shown in Figures 8a-c. Dysplastic foci with low CK7 expression but high nuclear TP53 expression were shown in Figures 8d-f. Dysplastic foci with both TP53 and CK7 high expression were shown in Figures 8g-i. When both TP53 $>8 \%$ and CK7 $>30 \%$ were used to diagnose dysplasia, the specificity achieved as high as $95 \%$. When either TP53 $>8 \%$ or CK7 $>30 \%$ was used to diagnose dysplasia, the sensitivity achieved was $82 \%$.

\section{Sensitivity Analysis}

Without $15(9 \%)$ indefinite for dysplasia cases in the non-dysplasia group, the cutoff values, model parameters, and receiver operating characteristic curves did not deviate from those of the initial model. For TP53, the new cutoff value was $6 \%$, sensitivity was $64 \%$, specificity was $89 \%$, and area under the curve was 0.77 (95\% CI: 0.69-0.84). For CK7, the cutoff remained $30 \%$, new sensitivity was $63 \%$, specificity was $74 \%$, and area under the curve was $0.68(0.59-0.76)$. For TP53 $>6 \%$ and CK7 $>30 \%$, the sensitivity was $36 \%$ and the specificity was $93 \%$. For TP53>6\% or CK7 $>30 \%$, the sensitivity was $83 \%$ and the specificity was $62 \%$. Only $1(1 \%)$ focus was reclassified from TP53 low expression group to TP53 high expression group because of the cutoff change from 8 to $6 \%$ for TP53. This sensitivity analysis further supported the robustness of our model in its potential application to daily practice, where the colon surveillance biopsy from inflammatory bowel disease shows a spectrum of changes from negative, indefinite for dysplasia, to dysplasia.

\section{Discussion}

Inflammatory bowel disease is an important risk factor for colorectal adenocarcinoma. Patients with inflammatory bowel disease usually undergo routine colonoscopic surveillance with biopsies to 

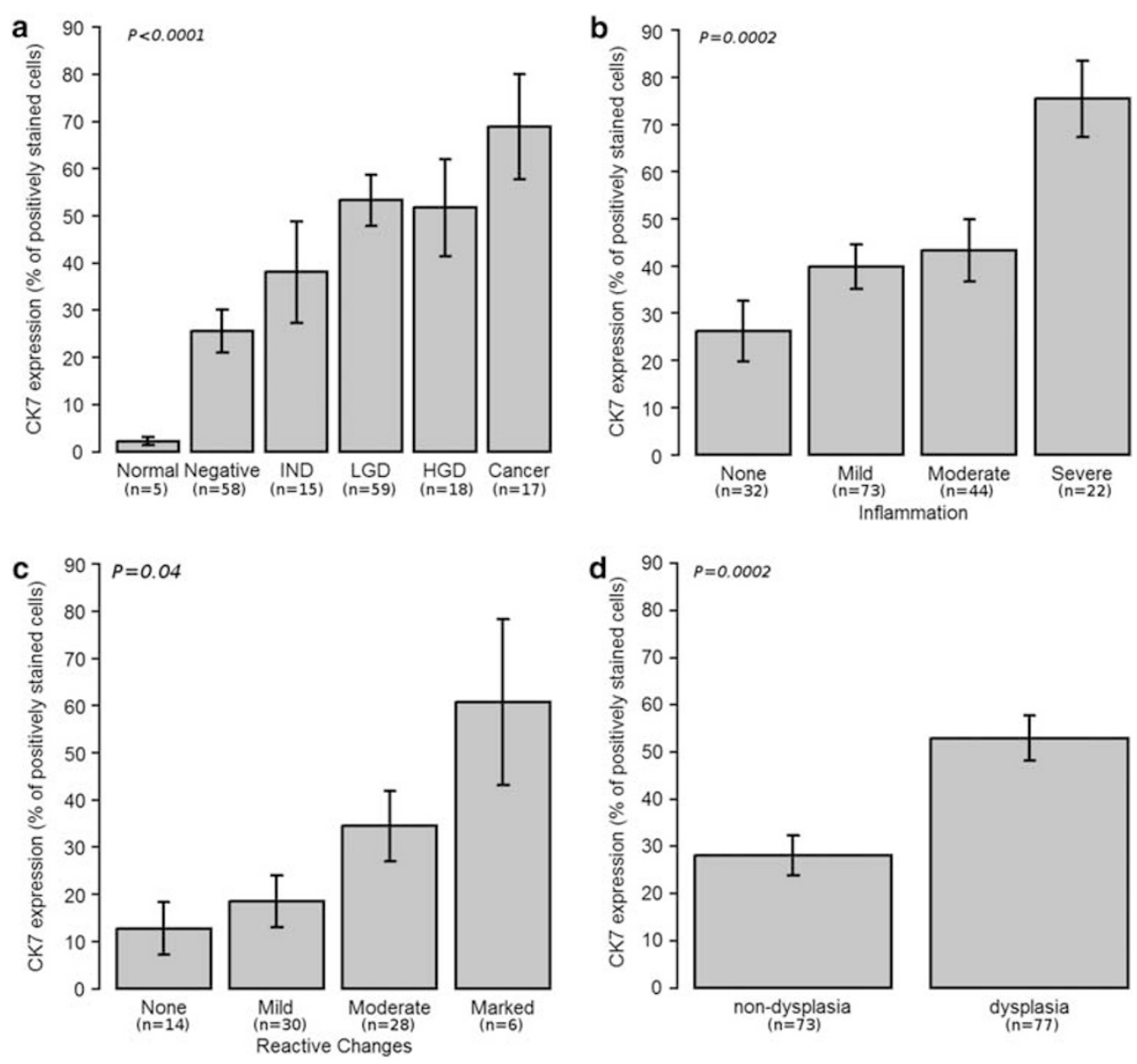

Figure 6 CK7 expression (\% of positively stained cells) and its relationship with histological neoplasia diagnosis (a), the presence of inflammation (b), reactive changes (c), and dysplasia diagnosis (d). HGD, high-grade dysplasia; IND, indefinite for dysplasia; LGD, lowgrade dysplasia. Error bar: 1 s.e.

Table 1 Logistic regression model for variables associated with the presence of dysplasia

\begin{tabular}{|c|c|c|c|c|c|c|}
\hline \multirow[t]{2}{*}{ Variable } & \multicolumn{3}{|c|}{ Full model } & \multicolumn{3}{|c|}{ Reduced model } \\
\hline & $\begin{array}{l}\text { Estimate } \\
(95 \% \text { CI) }\end{array}$ & $\begin{array}{l}\text { Odds ratio } \\
(95 \% \text { CI })\end{array}$ & $\mathrm{P}$-value & $\begin{array}{l}\text { Estimate } \\
(95 \% \text { CI) }\end{array}$ & $\begin{array}{l}\text { Odds ratio } \\
(95 \% \mathrm{CI})\end{array}$ & P-value \\
\hline TP53 & $0.028(0.005-0.05)$ & $1.03(1.01-1.05)$ & 0.02 & $0.038(0.021-0.055)$ & $1.04(1.02-1.06)$ & $<0.0001$ \\
\hline CK7 & $0.013(0.0004-0.026)$ & $1.01(1.0004-1.03)$ & 0.04 & $0.015(0.006-0.025)$ & $1.02(1.01-1.03)$ & 0.001 \\
\hline Inflammation (high/low) & $-1.5(-3.0-0.04)$ & $0.23(0.05-1.04)$ & 0.06 & - & - & - \\
\hline TP53 $\times$ inflammation & $0.026(-0.015-0.067)$ & $1.03(0.98-1.07)$ & 0.2 & - & - & - \\
\hline CK7 × inflammation & $0.0065(-0.015-0.028)$ & $1.01(0.98-1.03)$ & 0.6 & - & - & - \\
\hline TP53 $\times$ CK7 & $0.0001(-0.0004-0.0007)$ & $1.0001(1.0-1.001)$ & 0.6 & - & - & - \\
\hline
\end{tabular}

Abbreviation: CI, confidence interval.

Bold values show $P<0.05$.

search for dysplasia, a marker for the presence of synchronous or the development of metachronous colorectal adenocarcinoma. While histologic criteria for inflammatory bowel disease-associated dysplasia were proposed more than three decades ago, significant intra- and inter-observer's variability exists. Biomarkers, either in blood, stool, or tissue for the diagnosis of inflammatory bowel disease- associated neoplasia may potentially increase the screening accuracy.

Previous studies on tumorigenesis of inflammatory bowel disease-associated colonic adenocarcinoma showed many types of genetic abnormalities with one of them being mutation of TP53, a tumor-suppressor gene. This study showed that 44 $(57 \%)$ of inflammatory bowel disease-dysplasia had 
a

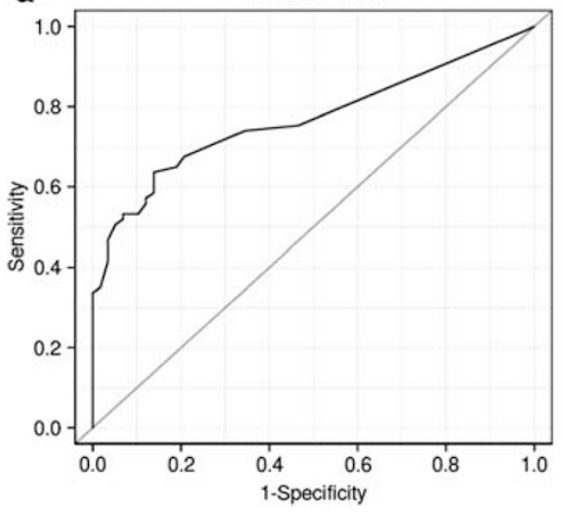

b

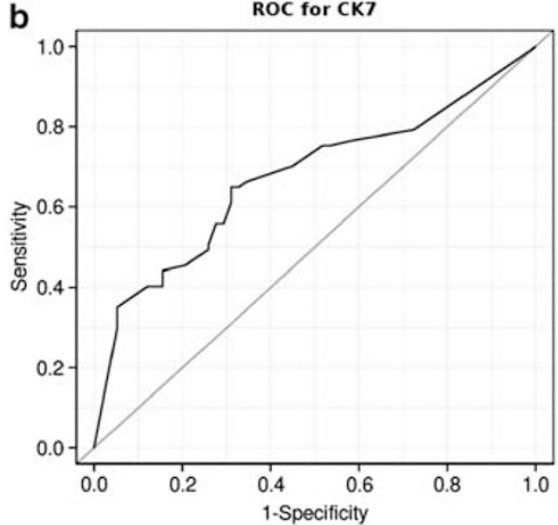

C

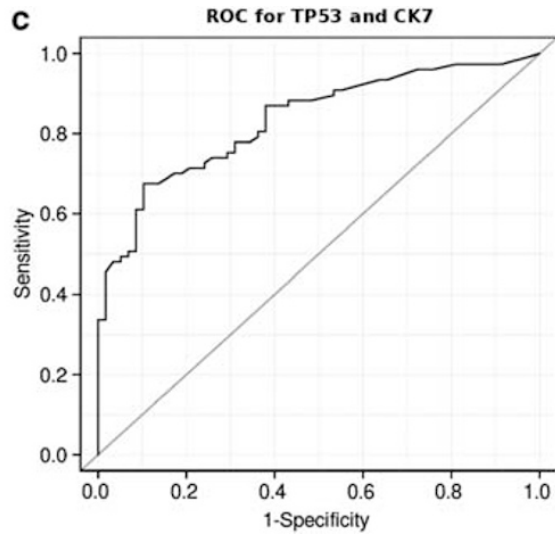

Figure 7 Receiver operating characteristic (ROC) curves of the logistic regression models with TP53 (a), CK7 (b), and a combination of TP53 and CK7 (c).

Table 2 Predictive value of TP53 and CK7 to the presence of dysplasia

\begin{tabular}{lcccccc}
\hline Variable & Cutoff & Sensitivity & Specificity & $P P V$ & $N P V$ & AUC \\
\hline TP53 & 8 & 0.6 & 0.87 & 0.83 & 0.67 & $0.75(0.66-0.82)$ \\
CK7 & 30 & 0.63 & 0.72 & 0.72 & 0.64 & $0.65(0.56-0.73)$ \\
TP53 and & $0.54^{\mathrm{b}}$ & 0.68 & 0.85 & 0.82 & 0.71 & $0.81(0.73-0.87)$ \\
CK7 & & & & & & \\
TP53 $>8$ & - & 0.36 & 0.95 & 0.88 & 0.58 & - \\
$\begin{array}{l}\text { and CK7 }>30 \\
\text { TP53 }>\text { 8 }\end{array}$ & - & 0.82 & 0.56 & 0.66 & 0.75 & - \\
or CK7 $>$ 30 & & & & & & \\
\hline
\end{tabular}

Abbreviations: AUC, area under the curve; NPV, negative predictive value; PPV, positive predictive value.

${ }^{\mathrm{a}}$ AUC (95\% confidence interval).

${ }^{\mathrm{b}}$ Cutoff for TP53 and CK7 was the probability of dysplasia predicted from the logistic regression model.

increased TP53 nuclear staining (defined as the presence of $8 \%$ of nuclei with intense staining), in line with several studies that have been shown that TP53 nuclear staining (defined as $\geq 5 \%$ of the total number of epithelial cells in a high-power field) occurred in approximately $50 \%$ of inflammatory bowel disease-associated neoplasia. ${ }^{15}$ Furthermore, this study clearly showed that TP53 nuclear staining was independent of inflammation or reactive changes, supporting that TP53 nuclear staining results from genetic mutation. This result suggested that TP53 may be diagnostically useful in biopsies with significant inflammation and reactive changes but worrisome features for dysplasia. In such cases, strong and diffuse nuclear staining for TP53 may aid a diagnosis of dysplasia. However, a negative TP53 staining result should not exclude the diagnosis of dysplasia.

CK7 is a cytokeratin with unknown biologic function. CK7 immunoreactivity has been routinely used in pathology practice to determine the possible origin of metastatic adenocarcinoma. It has been recently described in small bowel adenocarcinoma, ${ }^{16}$ pouch and peri-pouch adenocarcinoma, ${ }^{5}$ and inflammatory bowel disease-associated neoplasia in small series. ${ }^{6-8}$ However, CK7 immunoreactivity in inflammatory bowel disease-associated neoplasia has not been well investigated in a cohort with reasonable size. In this study, we included 39 colectomy specimens performed for inflammatory bowel disease-associated neoplasia and a total of 171 foci containing a spectrum of changes along the tumorigenesis of inflammatory bowel diseaseassociated neoplasia that were reviewed by three experienced gastrointestinal pathologists. Our study showed that CK7 expression was associated with progression of inflammatory bowel diseaseassociated neoplasia. The frequency of positive CK7 immunoreactivity in low-grade dysplasia, highgrade dysplasia, and cancer in our study was consistent with that reported in previous studies. ${ }^{6-8}$ Although patchy CK7 immunoreactivity associated with active epithelial inflammatory areas has been observed by Stenling et $a l^{8}$ our studies went one step further and found that CK7 immunoreactivity was associated with inflammation in the entire cohort and associated with reactive changes (but not inflammation) in the foci, which were negative or indefinite for dysplasia. Taken together, these results suggested that CK7 immunoreactivity may occur in two biological processes, true neoplasia and reactive/reparative process. In our studies, among 33 TP53 low expression dysplasia foci, 19 foci $(58 \%)$ had high expression of CK7, clearly indicating that there was a subset of dysplasia, which was CK7 positive but lack significant TP53 nuclear staining. The regulation of CK7 expression remains poorly understood. Although we clearly observed an upregulation of CK7 in inflammatory bowel disease-associated dysplasia, cancer, ${ }^{4}$ and pouch and peri-pouch adenocarcinoma, ${ }^{5}$ the mechanism leading to its upregulation during the tumorigenesis of inflammatory bowel disease-associated neoplasm remains unknown.

Inflammatory bowel disease-associated neoplasia is a continuous process. To predict which patient is 

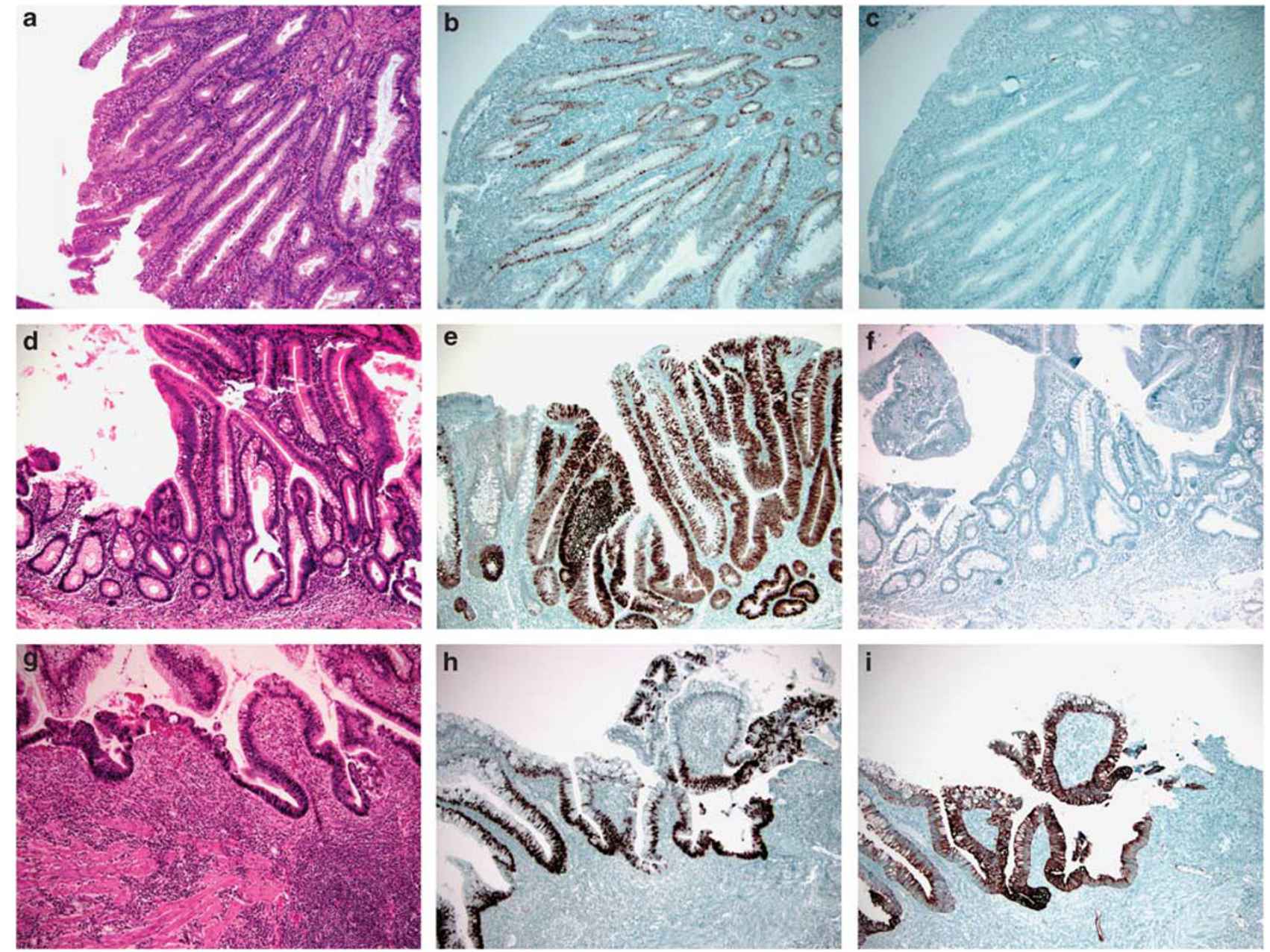

Figure 8 (a-c) A case of low-grade dysplasia with positive but relatively low TP53 nuclear staining and negative CK7 immunoreactivity (a, H\&E stain, $\times 100$; b, immunoperoxidase stain for TP53, $\times 100$; $\mathbf{c}$, immunoperoxidase stain for CK7, $\times 100)$. (d-f) A case of low-grade dysplasia with high TP53 nuclear staining but negative immunoreactivity for CK7 (d, H\&E stain, $\times 100$; e, immunoperoxidase stain for TP53, $\times 100$; f, immunoperoxidase stain for CK7, $\times 100$ ). (g-i) A case of low-grade dysplasia with high TP53 nuclear staining and immunoreactivity for CK7 (g, H\&E stain, $\times 100$; h, immunoperoxidase stain for TP53, $\times 100$; i, immunoperoxidase stain for CK7, $\times 100$ ).

likely to progress or have a fast progression to advanced neoplasia is difficult. A recently published paper suggested that TP53 nuclear staining and AMCAR immunoreactivity in biopsies with flat low-grade dysplasia and indefinite for dysplasia predicts progression to advanced inflammatory bowel disease-associated neoplasia in a time period of 19 months. ${ }^{15}$ It remains to be determined if CK7 immunoreactivity and TP53 nuclear staining (co-expression) predicts progression to advanced neoplasia in inflammatory bowel disease patients.

Finally, we evaluated the performance of TP53, CK7, and a combination of TP53 and CK7 in diagnosing inflammatory bowel disease-associated dysplasia. Although they are promising markers, the performance of these markers alone is far from perfect. 'Blindly' applying these markers without close correlation with H\&E histology may be misleading, as histologic evaluation remains the gold standard in pathology practice. However, in difficult cases, immunohistochemical stain for both TP53 and CK7 may be helpful. For example, in a severely inflamed biopsy with features worrisome for dysplasia, a strong and diffuse TP53 nuclear staining pattern would alert the pathologist for a diagnosis of dysplasia or at least prompt a consultation. Alternatively, in a quiescent colitis without significant reactive changes, a diffuse and strong CK7 immunoreactivity should also alert the pathologist for a diagnosis of dysplasia or at least prompt a consultation.

In summary, this study revealed that TP53 and CK7 immunoreactivity were correlated with inflammatory bowel disease-associated neoplasia. Nuclear staining of TP53 is independent of inflammation or reactive changes, but CK7 immunoreactivity was associated with reactive changes but independent of inflammation in the subset of negative or indefinite for dysplasia cases. Our results suggested that TP53 and CK7 immunoreactivity may be diagnostically 
useful to distinguish dysplasia from non-dysplasia in some diagnostically challenging cases from inflammatory bowel disease surveillance biopsies. In addition, the co-expression of CK7 and TP53 may be a potential marker to predict the progression of inflammatory bowel disease-associated neoplasia.

\section{Disclosure/conflict of interest}

The authors declare no conflict of interest.

\section{References}

1 Harpaz N, Polydorides AD. Colorectal dysplasia in chronic inflammatory bowel disease: pathology, clinical implications, and pathogenesis. Arch Pathol Lab Med 2010;134:876-895.

2 Elmunzer BJ, Higgins PDR, Kwon YM, et al. Jumbo forceps are superior to standard large-capacity forceps in obtaining diagnostically adequate inflammatory bowel disease surveillance biopsy specimens. Gastrointest Endosc 2008;68:273-278.

3 Ullman TA, Itzkowitz SH. Intestinal inflammation and cancer. Gastroenterology 2011;140:1807-1816.

4 Liu X, Goldblum JR, Zhao Z, et al. Distinct clinicopathologic features of inflammatory bowel diseaseassociated colorectal adenocarcinoma: in comparison to sporadic microsatellite stable and Lynch syndromerelated colorectal adenocarcinoma. Am J Surg Pathol 2012;36:1228-1233.

5 Jiang W, Shadrach B, Carver P, et al. Histomorphologic and molecular features of pouch and peri-pouch adenocarcinoma: a comparison to ulcerative colitisassociated adenocarcinoma. Am J Surg Pathol 2012; 36:1385-1394.

6 Tatsumi N, Kushima R, Vieth M, et al. Cytokeratin $7 / 20$ and mucin core protein expression in ulcerative colitis-associated colorectal neoplamsms. Virchows Arch 2006;448:756-762.
7 Levi GS, Harpaz N. Intestinal low-grade tubuloglandular adenocarcinoma in inflammatory bowel disease. Am J Surg Pathol 2006;30:1022-1029.

8 Stenling R, Lindberg J, Rutegard J, et al. Altered expression of CK7 and CK20 in preneoplastic and neoplastic lesions in ulcerative colitis. APMIS 2007; 115:1219-1226.

9 Baars JE, Vogelaar L, Wolfhagen FHJ, et al. A short course of corticosteroids prior to surveillance colonoscopy to decrease mucosal inflammation in inflammatory bowel disease patients: results from a randomized controlled trial. J Crohns Colitis 2010;4:661-668.

10 Itzkowitz S. Colon carcinogenesis in inflammatory bowel disease: applying molecular genetics to clinical practice. J Clin Gastroenterol 2003;36:S70-S74.

11 Gerrits MM, Chen M, Theeuwes M, et al. Biomarkerbased prediction of inflammatory bowel diseaserelated colorectal cancer: a case-control study. Cell Oncol (Dordr) 2010;34:107-117.

12 Marx A, Wandrey T, Simon P, et al. Combined alphamethylacyl coenzyme A racemase/p53 analysis to identify dysplasia in inflammatory bowel disease. Hum Pathol 2009;40:166-173.

13 Walsh SV, Loda M, Torres CM, et al. P53 and beta catenin expression in chronic ulcerative colitisassociated polypoid dysplasia and sporadic adenomas: an immunohistochemical study. Am J Surg Pathol 1999;23:963-969.

14 Riddell RH, Goldman H, Ransohoff DF, et al. Dysplasia in inflammatory bowel disease: standardized classification with provisional clinical applications. Hum Pathol 1983;14:931-968.

15 van Schaik F, Oldenburg B, Offerhaus GJA, et al. Role of immunohistochemical markers in predicting progression of dysplasia to advanced neoplasia in patients with ulcerative colitis. Inflamm Bowel Dis 2012;18: 480-488.

16 Chen ZME, Wang HL. Alteration of cytokeratin 7 and cytokeratin 20 expression profile is uniquely associated with tumorigenesis of primary adenocarcinoma of the small intestine. Am J Surg Pathol 2004;28: 1352-1359. 